\title{
The Effect of Personality Traits and Beliefs on the Relationship between Injury Severity and Subsequent Sport Risk-Taking among Adolescents
}

\author{
Linda Paquette ${ }^{1}$, Maggie Dumais ${ }^{2}$, Jacques Bergeron ${ }^{3}$ and Éric Lacourse ${ }^{4}$ \\ 1,2 Health Sciences Department, Université du Québec à Chicoutimi, Chicoutimi, Canada \\ ${ }^{3}$ Department of Psychology, Université de Montréal, Montréal, Canada \\ ${ }^{4}$ Department of Sociology, Université de Montréal, Montréal, Canada
}

Correspondence should be addressed to: Linda Paquette; linda_paquette@uqac.ca

Received date: 29 July 2014; Accepted date: 29 January 2015; Published date: 4 March 2016

Academic Editor: Gerri R. Hanten

Copyright @ 2016. Linda Paquette, Maggie Dumais, Jacques Bergeron and Éric Lacourse. Distributed under Creative Commons CC-BY 4.0

\begin{abstract}
In winter sports, the high injury rate among adolescents raises questions about the way they practice their sport. It also raises interrogations on the impact of an injury on their behavior on the slopes. The few studies that looked at the relationship between previous injury and subsequent risk-taking in sports ranged from no correlation (Kontos, 2004) to a positive correlation (Thomson et al., 2012). This study aimed to investigate the relationship between previous injuries and subsequent risk-taking among adolescent snowboarders and alpine skiers controlling for possible effects of beliefs related to risk-taking and personality traits. We analyzed cross-sectional data from 683 Canadian adolescents snowboarders and alpine skiers ( 367 boys, 316 girls), aged 14 to 17 years. Participants provided self-reported informations that evaluated risk-taking, impulsivity, sensation seeking and physical aggression. Different aspects of their sport practices, risk-related beliefs, and their sportsrelated injuries were assessed. Multivariate linear regression analysis showed that the severity of snowboarding- or alpine-skiing-related injuries that had occurred in the previous 12 months was associated with greater risk-taking, especially among impulsive adolescents. The regression model explained $57.4 \%$ of the variance of the risk-taking scale. The association between injury severity and risk-taking remained after controlling for sex, risk-related cognitive beliefs (perceived skills, risk estimation, and valorization of injuries), and personality traits (sensation seeking, impulsivity, and physical aggression). Injury might act as positive reinforcement for risk-taking among some adolescent snowboarders and alpine skiers.
\end{abstract}

Keywords: Risk-taking, adolescence, personality, injury

Cite this Article as: Linda Paquette, Maggie Dumais, Jacques Bergeron and Éric Lacourse (2016), "The Effect of Personality Traits and Beliefs on the Relationship between Injury Severity and Subsequent Sport Risk-Taking among Adolescents", Pediatrics Research International Journal, Vol. 2016 (2016),

Article ID 405500, DOI: 10.5171/2016.405500 


\section{Introduction}

Winter sports such as snowboarding and alpine skiing are widely practiced. There are an estimated 5.9 million snowboarders and 6.5 million alpine skiers in North America (SIA, 2010). These sports are associated with many injuries. An estimated 62,000 snowboarding-related injuries and 77,300 skiing-related injuries are treated annually in U.S. hospital emergency departments (Xiang, Kelleher, Shields, Brown and Smith, 2005). In Quebec, snowboarding is ranked fifth among sports that cause the most injuries, while alpine skiing ranks $12^{\text {th }}$ (Hamel and Tremblay, 2012). Head, neck, and trunk injuries from these sports are five times more prevalent in adolescents aged 12 to 17 years old than in adults aged 34 or older (Hagel et al., 2004). This high injury rate among adolescents raises concerns about the way they practice these sports.

Several studies have examined the relationship between previous risk-taking and subsequent injuries (Goulet, Régnier, Vallois and Ouellet, 2003; Turner, McClure, and Pirozzo, 2004; de Looze et al., 2011), but few investigators have studied the relationship between previous injury and subsequent risk-taking (Kontos, 2004). In snowsports (Thomson, Morton, Carlson and Rupert, 2012) and high risk sports (Woodman, Barlow, Bandura, Hill, Kupciw and MacGregor, 2013), studies showed a positive correlation between previous injury and subsequent risk-taking. A study of snowboarding also reported that previous injury predicts subsequent injury (Machold et al., 2000).

Initial beliefs and pre-existing personality traits might explain or influence the positive relationship between previous injury and subsequent risk-taking. For example, risk- and injury-related beliefs such as injury-risk perception (Siegel et al. 1994; Smith and Baxter, 2010), perceived skill level (Ruedl et al., 2010), and a positive attitude towards injury (Thorpe, 2004) have been associated with risktaking. Studies have also shown that personality traits such as sensation seeking
(Arnett, 1994), impulsivity (Lynam, and Miller, 2004), and aggression (Zuckerman and Kuhlman, 2000) are correlated with risk-taking. To the best of our knowledge, no study has examined the relationship between previous injury and subsequent risk-taking while controlling for these variables. We aimed to explore this relationship among adolescent snowboarders and alpine skiers, including possible moderating effects of these beliefs and personality traits.

\section{Definition of risk taking}

Risk-taking can be defined as engaging in a voluntary behavior that may lead to injury or death (Turner et al., 2004). Among adolescents, the relationship between previous risk-taking and subsequent injury is well established in various domains (de Looze et al., 2011) including car driving (Simms-Morton et al., 2011). Other studies have found a less consistent relationship between previous injuries and risk-taking in sports (Turner et al., 2004, Kontos, 2004). To date, studies on risk-taking in sports have been performed for sports that are considered risky (e.g., downhill racing), but the studies did not consider skill levels. In alpine sports, a study suggests that people who take more risks do not sustain more subsequent injuries, but they have higher skill levels (Cherpitel, Meyers, and Perrine, 1998; Goulet, Régnier, Valois and Ouellet, 2003). The same behavior may be risky for a beginner but not for an expert, due to their different skill levels. To address this difference, an intentional risktaking scale was developed and validated for snowboarding and alpine skiing. The validation study revealed a significant correlation between this risk-taking scale and several psychosocial correlates of risktaking, including sensation seeking, impulsivity and male sex (Paquette et al., 2009).

\section{Injuries and Subsequent Risk-Taking}

To the best of our knowledge, the only published longitudinal study specifically conducted on the relationship between previous sports injury and subsequent 
risk-taking in adolescents was conducted among soccer players, and it did not find any significant relationship between those two variables (Kontos, 2004). However, in this study risk-taking was assessed by looking at objective behaviors (e.g., diving headers) rather than intention to take risks. Among adults, other cross-sectional studies used past injuries as a predictive criterion proving the external validity of intentional risk-taking scales in alpine skiing and snowboarding (Thomson et al., 2012), and in high risk sports (Woodman et al., 2013). Both of these studies showed a positive correlation between past injury and subsequent self reported risk-taking. However, none of these researchers have considered the possibility that previous injury might have an impact on subsequent risk-taking, and none of them controlled for a possible interaction with other variables that could explain this relationship. According to Machold et al. (2000), snowboarders with a history of sports injury are 35\% more likely than uninjured snowboarders to get hurt again when snowboarding. The authors explain the results by a continued or increased risk-taking among injured snowboarders, but no risk-taking evaluation was made to support this interpretation. Similarly, a study of drivers who had been involved in road accidents that resulted in serious injuries found that after the accident, driving offences related to driving increased (Rajalin and Summala, 1997).

\section{Psychosocial Correlates of Risk Taking}

Since risk-taking after an injury might be influenced by initial conditions that have been identified in the literature as psychosocial correlates of risk-taking, these dimensions must be included in this study.

\section{Demographics}

Generally, men will take more risks in snowboarding and alpine skiing than women and they sustain more injuries (Hagel et al., 2004). Adolescents younger than 18 years old are also the most susceptible to alpine-snow-sport injuries, to exceed the speeds at which they feel in control and to consider themselves as risktakers (Bianchi, Brugger, Niemann and Cavegn, 2010). According to Fessler and coll (2013), young men take more risks because it enhances their conceptualized formidability. They named this association between risk-taking valorization and masculinity the "Crazy bastard hypothesis". From an evolutionary framework, the authors state that this would be an asset in finding more sexual partners and increase popularity.

\section{Personality traits}

Sensation seeking can be defined as a personality trait that characterizes individuals who are ready to take physical, financial, and social risks in order to seek intense and varied thrills (Zuckerman and Kuhlman, 2000). This trait was found to be positively correlated with risk-taking in alpine snow sports (Paquette et al., 2009).

Impulsivity is characterized by a tendency to act spontaneously and without deliberation (Bloch et al., 1997). Adolescents with high levels of impulsivity are two to eight times more likely than youth with low levels of impulsivity to engage in risky behaviors (Stanford et al., 1996). Impulsiveness has been linked with risk-taking in alpine snow sports (Paquette et al., 2009).

Zuckerman and Kuhlman (2000) observed that their Aggression-Hostility Personality scale values correlated positively with risktaking related to alcohol drinking, smoking, and unprotected sex. Physical aggression is also related to risk-taking in driving (Begg and Langley, 2004).

\section{Risk- and Injury-Related Beliefs}

A study among school-age children demonstrated a positive relationship between attribution of injuries to bad luck and engaging in risky behaviors (Morrongiello and Rennie, 1998). No study was conducted on this topic among adolescents older than 12 years, but similar results might be expected among them. 
Siegel et al (1994) observed a negative correlation between perceived risk and involvement in risky behaviors in sports. However, a study among soccer players found a positive correlation between these variables (Kontos, 2004). This might be explained by differences in the valorization of risk-taking. The glorification of injuries in snowboarding is an emerging trend. In sports-magazine articles, the snowboarders are often pictured showing their scars from serious injuries, which helps reinforce the idea that injuries are a sign of courage that people can take pride in (Thorpe, 2004). A qualitative study by Anderson (1999) reported that the snowboard culture values risk-taking. Snowboarders are indeed more likely to take risks than skiers (Paquette et al., 2009) and to get injured practicing their sport (Hagel et al., 2004).

Snowboarding injuries might enhance subsequent risk-taking due to the resulting feeling of pride. A recent study conducted among young adults skateboarders indicates that achieving one's best and the core value of freedom overweight the risk of injury associated with this sport. The results also indicate that injuries are accepted as a occupational hazard that reinforce their belonging to the skateboarding culture (Haines, Smith and Baxter, 2010). As skateboarding and snowboarding are similar sports, it might be expected that similar results will be found among adolescent snowboarders.

Other beliefs might be associated with risktaking. A study showed that alpine skiers and snowboarders who perceive themselves to have a high skill level are twice as likely to view themselves as risk takers compared with individuals who perceive themselves to have a lower skill level (Ruedl et al., 2010).

\section{Research Question and Hypotheses}

We sought to answer the following question: Do personality traits and riskand injury-related beliefs identified in the literature have a moderating influence on the relationship between injury severity and subsequent risk-taking among adolescent snowboarders and alpine skiers?

Based on the existing literature, we hypothesized that:

1) There is a positive relationship between the severity of an alpinesnow-sport injury sustained in the previous 12 months and the degree of subsequent risk-taking in adolescent snowboarders and alpine skiers.

2) Age, male sex, snowboarding, sensation seeking, impulsiveness, estimated risk of injury, fatalistic beliefs, valorization of injuries, and a high perceived skill level are positively associated with risktaking on the slopes.

\section{Method}

\section{Data Collection and Sample}

The data were collected during the 2006 ski season from French-speaking students in grades 9, 10, and 11 in two high schools located in Quebec, Canada. These two high schools were randomly selected from schools located within $5 \mathrm{~km}$ from a ski slope in the Quebec province, Canada. A 45minute questionnaire was administered during a class period. The first part of the questionnaire was completed by all students and then followed by second section specific to alpine snow sports. The administration of the questionnaire was supervised by a researcher and participants were assured of the confidentiality of their responses. The research project was approved by the ethics committee of the Université de Montréal.

The participation rate was $99 \%$. As the data collection was supervised by researchers in the class, the missing data is negligible below $5 \%$. Of the 1,021 students who agreed to participate, 999 completed the questionnaire. Of the total sample, 683 alpine sliding sports fans were selected for this study, including 316 girls and 367 boys. The participants were aged from 14 to 17 years $(M=15.53$ years). Of these, 187

Linda Paquette, Maggie Dumais, Jacques Bergeron and Éric Lacourse (2016), Pediatrics Research International Journal, DOI: 10.5171/2016.405500 
(27.4\%) had at least one injury that required the services of a health care professional in the past 12 months. Boys accounted for almost $60 \%$ of injuries. Among the injured, 58.3\% snowboarding adepts, $20.9 \%$ alpine skiing, and 20.9\% emerging sports ${ }^{1}$. Of all injured adolescents, $82 \%$ reported having been limited in their activities for several days following the injury, $10.8 \%$ were evacuated from the ski-resort by ambulance, $16.8 \%$ were hospitalized (at least one night at the hospital) and $11.8 \%$ reported having had at least two injuries requiring consultation with a health care professional. Table 1 shows the sample description.

\section{Instruments}

The Risk-Taking Scale in Snowboarding and Alpine Skiing (RISSKI, Paquette et al., 2009) assesses self-reported intentional reckless behaviors in alpine snow sports using six items (e.g., doing jumps or dangerous maneuvers for fun, looking for a thrill). In the current study, the Cronbach's alpha was 0.85 .

The Arnett Inventory of Sensation Seeking (AISS) scale (Arnett, 1994; French version, Cazenave and Paquette, 2010) assesses sensation seeking. The original English version consists of two scales composed of ten Likert-type items evaluating intensity seeking and novelty seeking. Cronbach's alpha values reported for the original AISS vary between 0.22 and 0.70 for the total score and for each subscale (Cazenave et Paquette, 2010). The French version, validated by a confirmatory factor analysis, was reduced to six Likert-type items in each scale. The Cronbach's alphas were 0.58 for the global scale, 0.56 for the intensity-seeking scale, and 0.36 for the novelty-seeking scale.

The French version of the Barratt Impulsiveness Scale (BIS-10; v.f. Baylé et al., 2000) assesses impulsivity using 34 Likert-type items. The Cronbach's alpha was 0.77 .

The Physical Aggression Scale measured the frequency of four aggressive behaviors: fist fighting, using physical force to dominate someone, using a weapon during a fight, and physically intimidating someone to get something (Tremblay, Pihl, Vitaro and Dobkin, 1994; Lacourse, Nagin, Tremblay, Vitaro and Claes 2003). The Cronbach's alpha was 0.71 .

We developed a questionnaire that evaluates risk- and injury-related beliefs using seven Likert separate items: 1) Association of pride with injuries; 2) The belief that injuries are signs of courage; 3 ) Estimation of risk of injury; 4) Perceived skill level; 5) The belief that reckless people are more popular; 6) Having more respect for reckless people; and 7) Believing that injuries are caused by fate or bad luck.

We developed a questionnaire including the type of sport, the number of years of experience in the sport and the frequency of sport practice. We created a composite winter sliding sport injury-severity index through consultation with sports professionals and researchers from the Ministère de l'Éducation, du Sport et du Loisir du Québec (MELS). As a positive correlation between previous injuries and subsequent risk-taking might only be true for less severe injuries, we controlled for the severity of the injury using this index. The index was based on answers to the following five questions:

1) Over the past 12 months, have you suffered an injury requiring consultation with a health professional? 2) If so, how often were you hurt? If you sustained several injuries, answer the following questions based on the most serious injury: 3) Were you evacuated by ambulance because of this injury? 4) Were you hospitalized overnight? 5) Were you limited in your activities for several days? Each item was binary coded (where $0=$ No, and $1=$ Yes) and a continuous scale was developed by adding the items. This resulted in a score of 0 to 5 , where a score of 0 indicated no injury, 1 indicated an injury without negative impact, 2 indicated at least one negative impact, etc. 


\section{Analyses}

Pearson's correlation analyses were conducted to study the association between all study variables. A multiple linear regression analysis was then performed on the variables that were significantly correlated with risk-taking. The hierarchical (blockwise entry) method was used to assess moderation effects. This method is recommended when there are known predictors of the measured outcome. The regression analysis was conducted in five steps, with known predictors entered first: injury-severity index (Block 1); demographics and variables related to sports (Block 2); personality traits (Block 3); risk- and injury-related cognition (Block 4); and interaction effects (Block 5). The noncategorical variables were transformed into $\mathrm{Z}$ scores to allow standardizing scales for comparison. To assess moderating effects, interactions were computed between the injury-severity index and sex; injury-severity index and personality traits; and injury-severity index and beliefs related to risk. Due to a high correlation between items related to the association of pride with injuries and the belief that injuries are signs of courage $(r=0.60)$, the mean of these two items was computed and incorporated into the regression model as a single variable.

\section{Results}

\section{Correlations}

Risk-taking was positively correlated with injury-severity index $(\mathrm{r}=0.32, p<0,001)$, male gender $(\mathrm{r}=0.43, p<0,001)$, years of experience $(\mathrm{r}=0.22, p<0,001)$, frequency of sports practice $(\mathrm{r}=0.51, p<0,001)$, being proud of injuries $(\mathrm{r}=0.22, p<0,001)$, believing that injuries are signs of courage $(\mathrm{r}=0.21, p<0,001)$, believing that reckless people are more popular $(\mathrm{r}=0.30, p<$ 0,001 ), having more respect for reckless people $(\mathrm{r}=0.30, p<0,001)$, believing that injuries result from bad luck or fate $(\mathrm{r}=$ $0.08, p<0,05$ ), having a high perceived skill level $(\mathrm{r}=0.52, p<0,001)$, estimation of injury risk ( $\mathrm{r}=0.34, p<0,001)$, impulsivity $(\mathrm{r}=0.22, p<0,001)$, novelty seeking $(\mathrm{r}=$ $0.10, p<0,05)$, intensity seeking $(\mathrm{r}=0.38, p$ $<0,001)$, and physical aggression $(\mathrm{r}=0.28$, $p<0,001)$. Table 2 shows the correlation matrix.

Table 1: Sample description $(\mathrm{N}=683)$

\begin{tabular}{|l|c|c|}
\cline { 2 - 3 } \multicolumn{1}{c|}{} & No injury & Injury \\
\cline { 2 - 3 } \multicolumn{1}{c|}{} & (last 12 months) & \begin{tabular}{c} 
( $\begin{array}{c}\text { 1 last 12 } \\
\text { months }\end{array}$ \\
\cline { 2 - 3 } \multicolumn{1}{c|}{}
\end{tabular} \\
\hline Age & $\mathrm{M}=15.49$ years & $\mathrm{M}=15.64$ years \\
\hline Sex & & \\
\hline Female & & $40.10 \%$ \\
\hline Male & $48.60 \%$ & $59.90 \%$ \\
\hline Type of sport & $51.40 \%$ & \\
\hline Alpine skiing & & $20.90 \%$ \\
\hline & $28.40 \%$ & $58.30 \%$ \\
\hline
\end{tabular}

Linda Paquette, Maggie Dumais, Jacques Bergeron and Éric Lacourse (2016), Pediatrics Research International Journal, DOI: 10.5171/2016.405500 


\begin{tabular}{|c|c|c|}
\hline Snowboard & & \\
\hline Emerging sports & $17.90 \%$ & $20.90 \%$ \\
\hline \multicolumn{3}{|l|}{ Frequency of sport practice } \\
\hline Between 1 and 3 times during the season & $23.80 \%$ & $10.70 \%$ \\
\hline Between 1 and 3 times per month & $23.20 \%$ & $11.20 \%$ \\
\hline Between 1 and 3 times per week & $37.30 \%$ & $32.60 \%$ \\
\hline More than 3 times per week & $15.10 \%$ & $45.50 \%$ \\
\hline Years of experience & $\mathrm{M}=5.40$ years & $\mathrm{M}=6.69$ years \\
\hline \multicolumn{3}{|l|}{ Injury severity } \\
\hline $\begin{array}{l}\text { Multiple injuries ( } \geq 2 \text { over the last } 12 \\
\text { months) }\end{array}$ & & $11.30 \%$ \\
\hline Evacuation in ambulance & & $10.80 \%$ \\
\hline Hospitalisation & & $16.80 \%$ \\
\hline Activity limitation for several days & & $82.20 \%$ \\
\hline
\end{tabular}

Table 2: Initial correlations between gender, age, sport related variables, personality traits, risk/injury related cognitions and risk taking on the slopes

\begin{tabular}{|c|c|c|c|c|c|c|c|c|c|c|c|c|c|c|c|c|c|c|c|c|c|}
\hline \multicolumn{22}{|l|}{ Table 2} \\
\hline Variables & 1 & 2 & 3 & 4 & 5 & 6 & 7 & 8 & 9 & 10 & 11 & 12 & 13 & 14 & 15 & 16 & 17 & 18 & 19 & 20 & 21 \\
\hline 1. Gender : male & -- & & & & & & & & & & & & & & & & & & & & \\
\hline 2. Age & 0,08 & -- & & & & & & & & & & & & & & & & & & & \\
\hline 3. Type of sport : alpine skiing & $-0,11$ & 0,00 & -- & & & & & & & & & & & & & & & & & & \\
\hline 4. Type of sport : snowboard & 0,07 & $-0,01$ & $-0,66$ & -- & & & & & & & & & & & & & & & & & \\
\hline 5. Type of sport : emerging sports & 0,12 & 0,01 & $-0,29$ & $-0,53$ & - & & & & & & & & & & & & & & & & \\
\hline 6. Years of experience & 0,03 & 0,09 & 0,41 & $-0,41$ & 0,06 & -- & & & & & & & & & & & & & & & \\
\hline 7. Practice frequency & 0,16 & $-0,08$ & $-0,02$ & 0,00 & 0,02 & 0,37 & -- & & & & & & & & & & & & & & \\
\hline 8. Injury over the past 12 monts $\geq 1$ & 0,08 & 0,07 & $-0,08$ & 0,04 & 0,03 & 0,15 & 0,29 & - & & & & & & & & & & & & & \\
\hline 9. Injury severity index & 0,09 & 0,06 & $-0,05$ & 0,01 & 0,05 & 0,14 & 0,27 & 0,90 & -- & & & & & & & & & & & & \\
\hline 10. «We can be proud of our injuries» & 0,16 & $-0,03$ & $-0,01$ & $-0,01$ & 0,02 & 0,01 & 0,01 & 0,08 & 0,08 & -- & & & & & & & & & & & \\
\hline 11. «Injuries are signs of courage» & 0,17 & $-0,07$ & $-0,04$ & 0,03 & 0,04 & $-0,06$ & $-0,01$ & 0,04 & 0,04 & 0,60 & -- & & & & & & & & & & \\
\hline 12. "Those who take more risks are more popular» & 0,34 & $-0,05$ & $-0,12$ & 0,08 & 0,03 & $-0,04$ & 0,09 & 0,10 & 0,10 & 0,15 & 0,21 & -- & & & & & & & & & \\
\hline 13. «I have more respect for reckless people» & 0,26 & $-0,04$ & $-0,09$ & 0,08 & 0,01 & $-0,05$ & 0,12 & 0,08 & 0,06 & 0,21 & 0,29 & 0,37 & -- & & & & & & & & \\
\hline 14. «Injuries are caused by fate or bad luck» & 0,03 & 0,06 & $-0,01$ & 0,04 & $-0,04$ & $-0,06$ & 0,00 & 0,00 & 0,01 & 0,11 & 0,08 & 0,08 & 0,06 & -- & & & & & & & \\
\hline 15. Level of perceived skill & 0,22 & 0,03 & 0,14 & $-0,17$ & 0,06 & 0,57 & 0,63 & 0,24 & 0,24 & 0,05 & 0,01 & 0,12 & 0,04 & $-0,02$ & -- & & & & & & \\
\hline 16. Risk of injury estimation & 0,13 & 0,05 & $-0,14$ & 0,10 & 0,04 & 0,03 & 0,20 & 0,23 & 0,25 & 0,11 & 0,12 & 0,10 & 0,10 & $-0,01$ & 0,14 & -- & & & & & \\
\hline 17. BIS-10 impulsivity & 0,07 & 0,02 & $-0,15$ & 0,10 & 0,03 & $-0,03$ & 0,04 & 0,06 & 0,08 & 0,14 & 0,15 & 0,11 & 0,15 & 0,10 & 0,00 & 0,10 & -- & & & & \\
\hline 18. AISS novelty seeking & $-0,03$ & 0,09 & 0,02 & $-0,01$ & $-0,01$ & 0,06 & $-0,02$ & 0,04 & 0,05 & 0,09 & 0,02 & $-0,01$ & $-0,02$ & 0,07 & 0,03 & 0,10 & 0,14 & -- & & & \\
\hline 19. AISS intensity seeking & 0,35 & 0,05 & $-0,10$ & 0,03 & 0,08 & $-0,03$ & 0,07 & 0,14 & 0,13 & 0,27 & 0,19 & 0,21 & 0,19 & 0,05 & 0,08 & 0,07 & 0,26 & 0,23 & - & & \\
\hline 20. Physical aggression & 0,31 & $-0,03$ & $-0,11$ & 0,04 & 0,07 & 0,01 & 0,02 & 0,08 & 0,12 & 0,26 & 0,14 & 0,14 & 0,19 & 0,09 & 0,10 & 0,10 & 0,18 & 0,06 & 0,31 & -- & \\
\hline 21. Risk-taking scale & 0,43 & $-0,01$ & $-0,14$ & 0,07 & 0,07 & 0,22 & 0,51 & 0,28 & 0,32 & 0,22 & 0,21 & 0,30 & 0,30 & 0,08 & 0,52 & 0,34 & 0,22 & 0,10 & 0,38 & 0,28 & -- \\
\hline
\end{tabular}




\section{Regression Analysis}

The results of the regression analyses are shown in Table 3. The adjusted $\mathrm{R}^{2}$ indicates that the final model explains $57.4 \%$ of the risk-taking scale variance. The fifth model was selected because it accounted for the biggest amount of the risk-taking scale variance. The variables contributing to the final model were severity of injury $(\beta=$ $0.07, \mathrm{p}<0.01)$, male gender $(\beta=0.17, \mathrm{p}<$ $.001)$, snowboarding $(\beta=0.10, p<0.01)$, practice frequency $(\beta=0.26, p<0.001)$, intensity seeking $(\beta=0.15, \mathrm{p}<0.001)$, impulsivity $(\beta=0.09, \mathrm{p}<0.05)$, physical aggression $(\beta=0.06, p<0.05)$, high perceived skill level $(\beta=0.24, p<0.001)$, estimation of injury risk $(\beta=0.16, p<$ $0.05)$, having more respect for reckless people $(\beta=0.09, p<0.001)$, and believing that reckless people are more popular $(\beta=$ $0.09, \mathrm{p}<0.01$ ).
The preliminary regression analysis showed that the severity of injury was moderately correlated with risk-taking $(\beta=$ $0.31, p<0.001$ ), but when personality traits and beliefs related to risks and injuries were included in the regression model, this relationship decreased and it was lower in the final model $(\beta=0.07, \mathrm{p}<$ 0.01 ). An interaction effect was also found between injury-severity index and impulsivity $(\beta=0.07, \mathrm{p}<0.05)$. As shown in Figure 1, the correlation between risktaking and injury severity was higher among participants with high impulsivity (+1 SD) than in those with low impulsivity $(-1 \mathrm{SD})$. The contribution of the interaction effect between injury severity and impulsivity was small. However, the variables contributing most strongly to the model were, in order of decreasing importance: practice frequency $(\beta=.26, \mathrm{p}<$ $0.001)$, high perceived skill level $(\beta=0.24$, $\mathrm{p}<0.001)$, and male sex $(\beta=0.17, \mathrm{p}<$ 0.001 ). 


\begin{tabular}{|c|c|c|c|c|c|c|c|c|c|c|c|c|c|c|c|c|c|c|c|c|c|c|}
\hline \multirow[t]{2}{*}{ Variables } & \multicolumn{6}{|c|}{ Model 1} & \multicolumn{3}{|c|}{ Model 2} & \multicolumn{5}{|c|}{ Model 3} & \multicolumn{3}{|c|}{ Model 4} & \multicolumn{5}{|c|}{ Model 5} \\
\hline & $\mathbf{M}^{\mathrm{a}}$ & (SD) & B & $\beta$ & $p$ & $95 \%$ CI & B & $\beta$ & $p$ & $95 \% \mathrm{CI}$ & B & $\beta$ & $p$ & $95 \% \mathrm{CI}$ & B & $\beta$ & $\mathbf{p}$ & $95 \%$ CI & B & $\beta$ & $p$ & $95 \% \mathrm{CI}$ \\
\hline Injury severity index & 0.66 & 1.20 & 0.312 & 0.308 & $* * *$ & [0.236- 0.388$]$ & 0.162 & 0.160 & $* * * *$ & [0.099-0.225] & 0.123 & 0.122 & **** & [0.064-0.183] & 0.081 & 0.079 & $* *$ & {$[0.025-0.137]$} & 0.067 & 0.066 & $*$ & [0.010-0.124] \\
\hline \multicolumn{23}{|l|}{ Demographics } \\
\hline Sex & 1.46 & 0.50 & & & & & 0.689 & 0.345 & **** & {$[0.568-0.810]$} & 0.503 & 0.252 & ${ }^{* * * *}$ & {$[0.376-0.629]$} & 0.333 & 0.167 & $* * *$ & {$[0.209-0.456]$} & 0.330 & 0.166 & $* * *$ & {$[0.207-0.453]$} \\
\hline \multicolumn{23}{|l|}{ Sport related variables } \\
\hline Snowboard vs alpine skiing & 0.55 & 0.50 & & & & & 0.326 & 0.163 & $* * * *$ & {$[0.165-0.487]$} & 0.254 & 0.127 & $* *$ & {$[0.100-0.407]$} & 0.213 & 0.107 & $* *$ & {$[0.070-0.357]$} & 0.204 & 0.102 & ** & {$[0.061-0.347]$} \\
\hline Emerging sports vs alpine skiing & 0.19 & 0.39 & & & & & 0.254 & 0.101 & ** & {$[0.070-0.438]$} & 0.195 & 0.078 & * & {$[0.020-0.369]$} & 0.158 & 0.063 & & {$[-0.004-0.320]$} & 0.147 & 0.059 & & {$[-0.015-0.309]$} \\
\hline Years of experience & 5.76 & 3.84 & & & & & 0.095 & 0.096 & $*$ & {$[0.022-0.168]$} & 0.095 & 0.096 & $* *$ & {$[0.026-0.165]$} & 0.020 & 0.020 & & {$[-0.051-0.090]$} & 0.019 & 0.020 & & {$[-0.051-0.090]$} \\
\hline Sport practice frequency & 2.63 & 1.05 & & & & & 0.387 & 0.387 & **** & {$[0.320-0.455]$} & 0.399 & 0.399 & **** & {$[0.335-0.463]$} & 0.247 & 0.247 & $* * *$ & {$[0.178-0.317]$} & 0.256 & 0.256 & $* * *$ & {$[0.186-0.325]$} \\
\hline \multicolumn{23}{|l|}{ Personality } \\
\hline AISS novelty seeking & 27.05 & 4.15 & & & & & & & & & 0.073 & 0.073 & * & {$[0.16-0.131]$} & 0.052 & 0.052 & & {$[-0.002-0.106]$} & 0.052 & 0.052 & & {$[-0.001-0.105]$} \\
\hline AISS intensity seeking & 26.89 & 4.696 & & & & & & & & & 0.163 & 0.164 & **** & {$[0.098-0.227]$} & 0.149 & 0.150 & $* * *$ & {$[0.088-0.210]$} & 0.151 & 0.152 & $* * *$ & {$[0.090-0.212]$} \\
\hline BIS-10 impulsivity & 51.38 & 14.35 & & & & & & & & & 0.107 & 0.108 & $* * *$ & {$[0.049-0.165]$} & 0.086 & 0.087 & *** & {$[0.032-0.141]$} & 0.088 & 0.089 & $* *$ & {$[0.034-0.142]$} \\
\hline Physical agression scale & 5.24 & 1.80 & & & & & & & & & 0.099 & 0.100 & $* *$ & {$[0.039-0.159]$} & 0.061 & 0.062 & * & {$[0.004-0.119]$} & 0.063 & 0.064 & * & {$[0.006-0.120]$} \\
\hline \multicolumn{23}{|l|}{ Risk and injury related cognitions } \\
\hline Perceived skill level & 2.25 & 0.70 & & & & & & & & & & & & & 0.245 & 0.245 & $* * *$ & {$[0.168-0.322]$} & 0.242 & 0.241 & $* * *$ & {$[0.165-0.318]$} \\
\hline Injury risk estimation & 2.82 & 0.88 & & & & & & & & & & & & & 0.160 & 0.160 & $* * *$ & {$[0.105-0.215]$} & 0.160 & 0.160 & $* * *$ & {$[0.105-0.215]$} \\
\hline «Injuries are signs of courage/We can be proud of our injuries» & 1.69 & 0.93 & & & & & & & & & & & & & 0.043 & 0.043 & & {$[-0.014-0.099]$} & 0.039 & 0.039 & & {$[-0.017-0.096]$} \\
\hline «I have more respect for reckless people» & 2.11 & 1.35 & & & & & & & & & & & & & 0.099 & 0.098 & *** & {$[0.040-0.159]$} & 0.092 & 0.091 & ** & {$[0.033-0.152]$} \\
\hline «Injuries are caused by fate or bad luck» & 2.75 & 1.25 & & & & & & & & & & & & & 0.009 & 0.009 & & {$[-0.045-0.062]$} & 0.012 & 0.012 & & {$[-0.041-0.065]$} \\
\hline «Those who take more risks are more popular» & 2.79 & 1.45 & & & & & & & & & & & & & 0.081 & 0.082 & ** & {$[0.023-0.140]$} & 0.084 & 0.085 & *** & {$[0.026-0.143]$} \\
\hline \multicolumn{23}{|l|}{ Interaction } \\
\hline \multirow[t]{5}{*}{ Impulsivity*Injury severity index } & 0.08 & 1.03 & & & & & & & & & & & & & & & & & 0.062 & 0.066 & * & {$[0.012-0.113]$} \\
\hline & Con & stant $=$ & & -0.002 & & & & -0.603 & & & & -0.454 & & & & 0.440 & & & & 0.327 & & \\
\hline & & $R=$ & & 0.308 & & & & 0.663 & & & & 0.712 & & & & 0.763 & & & & 0.766 & & \\
\hline & & $R^{2}=$ & & 0.095 & & & & 0.440 & & & & 0.507 & & & & 0.582 & & & & 0.586 & & \\
\hline & Adjust & $e d R^{2}=$ & & 0.093 & & & & 0.434 & & & & 0.499 & & & & 0.571 & & & & 0.574 & & \\
\hline
\end{tabular}

BIS-10. Barratt Impulsiveness Scale; AISS. Arnett Inventory of Sensation Seeking

$* p<0.05 . * * p<0.01 . * * * p<0.001$.

${ }^{a}$ Original means and standard deviations are presented even if data were transformed in $\mathrm{Z}$ score to enable the equivalence of scales for

comparison. 


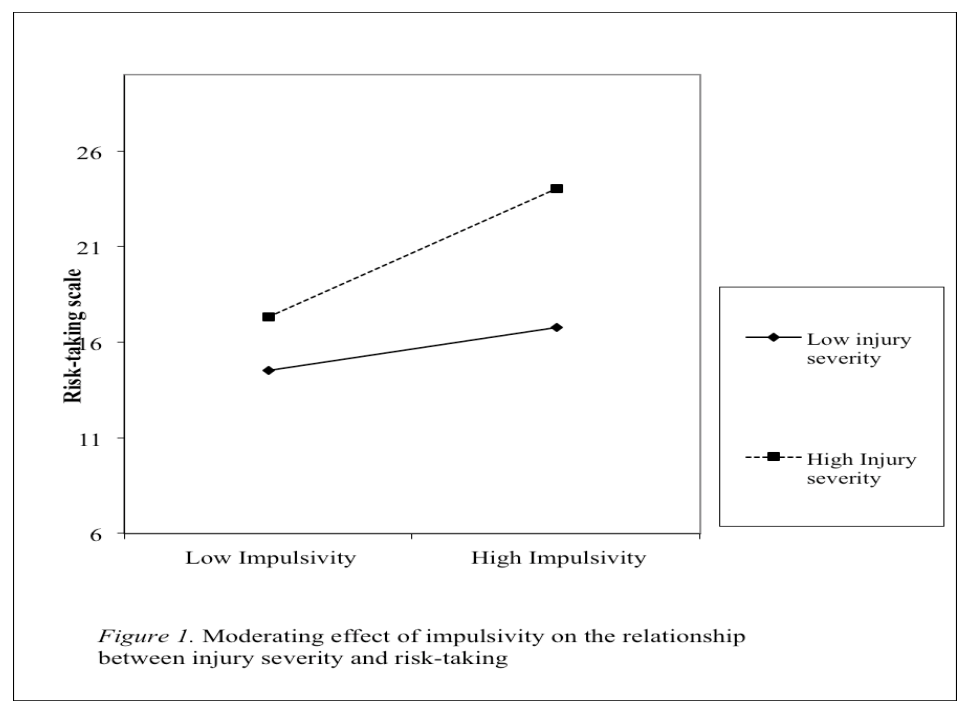

Figure 1: Moderating effect of impulsivity on the relationship between injury severity and risk-taking

\section{Discussion}

This study aimed to verify the link between the severity of an alpine sports injury sustained in the previous 12 months and risk-taking in snowboarding and alpine skiing, including possible moderating effects from personality traits and beliefs. To the best of our knowledge, this is the first study to include a wide range of psychosocial correlates of risk-taking that have been identified in the literature. It yielded a final regression model that explains a high percentage of the risktaking-scale variance (57.4\%).

In agreement with previous studies, the results showed that risk-taking in snowboarding and alpine skiing was significantly associated with male gender (Ruedl and al. 2010; Fessler et al., 2013), seeking intense sensation (Paquette et al., 2009), impulsivity (Lynam, and Miller, 2004), and physical aggression (Begg and Langley, 2004), which supports the hypothesis that risk-taking in sports is related to personality factors. Age was not related to the degree of risk-taking, possibly because of the narrow age range in this sample (14 to 17 years).
Beliefs are also associated with greater risk-taking. In agreement with work by Kontos (2004) and Goulet et al. (2003), a high level of perceived skill was linked with greater risk-taking. The estimated risk of injury was also positively associated with risk-taking, in agreement with the study by Kontos (2004). These latter results, however, are inconsistent with the negative relationship between risk assessment and risk-taking reported by studies conducted in other areas (Siegel et al., 1994). These differences may be due to a greater appreciation of risk-taking in sports (Thorpe, 2004; Haines et al., 2010), whereas in risky behaviors associated with civil disobedience, risk-taking is devalued. This positive attitude toward risk-taking in sports culture is also supported by the positive relationships between risk-taking and believing that reckless people are more popular, and respecting people who take greater risks.

In contrast with the results of Morrongiello and Rennie (1998), the initial correlation between fatalistic beliefs and risk-taking was low, and fatalistic beliefs did not contribute significantly to the final model. This might be explained by the correlation between having fatalistic beliefs and being proud of injuries. 
The initial regression analyses indicated that injury severity was associated with greater risk-taking, and this relationship contributed to the final model, even when sensation seeking and impulsivity were added to the model. These results are consistent with those of Machold et al. (2000), who noted that snowboarders who had a sports-related injury were $35 \%$ more likely to sustain subsequent snowboarding injuries. These results are also congruent with those of Rajalin and Summala (1997) in studies of driving, which showed that drivers who had an accident leading to a severe injury were more likely to drive dangerously than drivers who were not involved in an accident. The positive relationship between injury severity and subsequent risk-taking on the slopes may be due to the reinforcement of risk-taking in the winter-sliding-sport culture (Anderson, 1999; Thorpe, 2004; Haines et al., 2010). It may also be explained by the biological development of the adolescent's brain, making it more reactive to the dopaminergic rewards system in the stratium region of the brain and less active in the prefrontal cortex region, more specifically the inferior frontal gyrus, associated with the update of negative errors in the risks estimation (Moutsiana, Garrett, Clarke, Lotto, Blakemore \& Sharot, 2013). The rewards associated with risktaking and injuries might then overcome the negative aspects of injuries in adolescent snowboarders and alpine skiers for neurodevelopmental reasons.

Our analysis revealed a low interaction effect between impulsivity and injury severity, which suggests that impulsivity has a moderating effect on the relationship between injury severity and subsequent risk-taking. Among impulsive teens, the relationship between risk-taking and injury severity was stronger than among those who were less impulsive, which suggests that they may value injuries more. Consistent with this interpretation, in the initial correlation analysis, impulsivity was positively linked to the belief that one can be proud of one's injuries, the belief that risk-takers are more popular, and the practice of snowboarding rather than skiing. Personality traits would thus be associated with choosing activities involving a risk-taking culture and having values related to an appetite for risk, consistent with the results of qualitative studies by Anderson (1999), Thorpe (2004) and Haines et al. (2010).

The severity of an injury also seemed to share a common variance with personality traits and beliefs related to risk and injury. Indeed, when adding personality traits and beliefs to the regression model, the relationship between the severity of the injury and risk-taking decreased. Although this cross-sectional study was not designed to assess how these traits and beliefs affect the link between injury severity and risktaking, this study suggests that having traits of impulsivity, sensation seeking and physical aggression likely increases the risk of injury in sports. It is also conceivable that beliefs are favorable to risk and injury might predict the occurrence of an injury.

The results suggest that some adolescent snowboarders and alpine skiers have a positive attitude toward injuries. Among these individuals, becoming injured may act as a positive reinforcement to taking more risks, due to associations that are already in place before the injury (i.e., that risk-taking is a way to become popular and earn respect from their peers). The study also suggests that impulsive adolescents may take more risks after an injury. Thus, due to different personality traits and beliefs, the impact of an injury will vary from one individual to another.

\section{Study Limitations}

Because of the cross-sectional design of this study, it was not possible to determine a causal association between injury severity and risk-taking. We also considered the occurrence of an injury during the previous 12 months, since it may be difficult for individuals to recollect the exact times of their accidents. Another limitation of the study is the self reported nature of the questionnaires. A prospective, longitudinal study with observational data is needed to rigorously demonstrate the moderating or mediating effect of beliefs 
and personality traits related to risk-taking on the slopes, before and after an injury.

\section{Conclusion}

The results support the hypothesis that personality factors are at work in risky decision making following an injury, and an injury may act as positive reinforcement of risk-taking among some adolescent snowboarders and alpine skiers. This study included a wide-range of psychosocial correlates of risk-taking that may be useful in an injury-prevention perspective. An application of the results could be an injury-prevention campaign elaborated in order to reduce the positive image (courage, pride) associated with sports related injuries.

\section{Acknowledgement}

We would like to thank the Fonds de recherche en santé du Québec (FRSQ) the Ministère de l'éducation, du loisir et du sport du Québec (MELS) and the Université du Québec à Chicoutimi for the funding of this study.

\section{Notes:}

${ }^{1}$ Emerging sports : Twin tips $(\mathrm{N}=66)$, Snowblade ( $\mathrm{N}=39)$, Acrobatic skiing $(\mathrm{N}=15)$, Snowskate $(\mathrm{N}=7), 3$-ski $(\mathrm{N}=3)$.

\section{References}

1) Anderson, K. L. (1999) "Snowboarding: The construction of gender in an emerging sport," Journal of Sports and Social Issues, 23 (1), 55-79.

2) Arnett, J. (1994) "Sensation seeking: A new conceptualisation and a new scale," Personality and Individual Differences, 16 (2), 289-296.

3) Baylé, F. J., Bourdel, M. C., Caci, H., Gorwood, P., Chignon, J. M., Adès, J., and Loo, H. (2000) "Structure factorielle de la traduction française de l'échelle d'impulsivité de Barratt (BIS-10)," Revue canadienne de psychiatrie, 45, 156-165.
4) Bloch, H., Dépret, E., Gallo, A., Garnier, PH., Gineste, M.-D., Leconte, P., Le Ny, J.-F., Postel, J., Reuchlin, M., \& Cassis, D. (1997) Dictionnaire fondamental de la psychologie. Larousse : Paris.

5) Begg, D. J., and Langley, J. D. (2004) "Identifying predictors of persistent nonalcohol or drug-related risky driving behaviors," Accident Analysis and Prevention, 36, 1067-1071.

6) Bianchi, G., Brugger, O., Niemann, S., and Cavegn, M. (2011) "Helmet use and self-reported risk-taking in skiing and snowboarding," Journal of ASTM International, 8 (1), 1-7.

7) Cazenave, N., and Paquette, L. (2010) "L'Arnett Inventory of Sensation Seeking (AISS): validation et évaluation psychométrique chez une population de jeunes étudiants français," Revue l'Encéphale, 36 (5), 366-372.

8) Cherpitel, C. J., Meyers, A. R., Perrine, M. W. (1998) "Alcohol consumption, sensation seeking and ski injury: A casecontrol study," Journal of Studies on Alcohol, 59 (2), 216-221.

9) De Looze, M., Pickett, W., Raaijmakers, Q., Kuntsche, E., Hublet, A., Gabhainn, S. N., Bjarnsason, T., Molcho, M., Vollebergh, W., and ter Bogt., T. (2012) "Early risk behaviors and adolescent injury in 25 european and north American countries : a cross-national consistent relationship," Journal of early adolescence, 32 (1), 104125.

10) Fessler, D. M. T., Tiokhin, L. B., Holbrook, C., Gervais, M. M., and Snyder, J. K. (2013) "Foundations of the Crazy Bastard Hypothesis: Nonviolent physical risk-taking enhances conceptualized formidability," Evolution and human behavior, Online first publication. http://dx.doi.org/10.1016/j.evolhumbehav .2013.09.003

11) Goulet, C., Régnier, G., Valois, P., and Ouellet, G. (2003) "Injuries and risk-taking in alpine skiing. In Johnson et al. (Eds.)," 
Skiing Trauma and Safety, vol. 13, ASTM 1397, 139-146.

12) Hagel, B, E., Goulet, C., Platt, R. W., and Pless, I. B. (2004) "Injuries among skiers and snowboarders in Quebec," Epidemiology, 15, 279-285.

13) Haines, C., Smith, T. M., and Baxter, M. F. (2010) "Participation in the risk-taking occupation of skateboarding," Journal of Occupational Science, 17 (4), 239-245.

DOI: $10.1080 / 14427591.2010 .9686701$

14) Hamel, D., and Tremblay, B. (2012) "Étude des blessures subies au cours de la pratique d'activités récréatives et sportives au Québec en 2009-2010". Ministère de l'Éducation, du Sport et du Loisir du Québec (MELS). Research report. TroisRivières.

15) Kontos, A. P. (2004) "Perceived risk, risk-taking, estimation of ability and injury among adolescent sport participants," Journal of Pediatric Psychology, 29 (6), 447455.

16) Lacourse, É., Nagin, D., Tremblay, R., Vitaro, F., and Claes, M. (2003) "Developmental trajectories of boys delinquent group membership and facilitation of violent behaviors during adolescence," Development and Psychopathology, 15, 183-197.

17) Lynam, D. R., and Miller, J. D. (2004) "Personality pathways to impulsive behavior and their relations to deviance," Journal of Quantitative Criminology, 20, 319-341.

18) Machold, W., Kwasny, O., Gabler, P., Kolonja, A., Reddy, B., Bauer, E., and Lehr, S. (2000) "Risk of injury through snowboarding," Journal of Trauma: Injury Infection and Critical Care, 48 (6), 11091114.

19) Morrongiello, B.A., and Rennie, $H$. (1998) "Why do boys engage in more risktaking than girls? The role of attributions, beliefs, and risk appraisals," Journal of Pediatric Psychology, 23 (1), 33-43.
20) Moutsiana, C., Garrett, N., Clarke, R. C., Lotto, R. B., Blakemore, S.-J., \& Sharot, T. (2013). Human development and the ability to learn from bad news. Procedings of the National Academy of Sciences of the United States, 110(41), 16396-16401.

21) Paquette, L., Lacourse, E., and Bergeron, J. (2009) "Construction d'une échelle de prise de risques et validation auprès d'adolescents pratiquant un sport alpin de glisse," Canadian Journal of Behavioral Science, 41 (3), 133-142.

22) Rajalin, S., and Summala, H. (1997) "What surviving drivers learn from a fatal road accident," Accident Analysis and Prevention, 29, 277-283.

23) Ruedl G., Pocecco E., Sommersacher, R., Gatterer, H., Kopp, M., Nachbauer, W., and Burtscher, M. (2010) "Factors associated with self-reported risk-taking behaviour on ski slopes," British Journal of Sports Medicine, 44, 204-206.

24) Siegel, A. W., Cousins, J. H., Rubovits, D. S., Parsons, J. T., Lavery, B., and Crowley, C. L. (1994) "Adolescents' perceptions of the benefits and risks of their own risk-taking," Journal of Emotional and Behavioral Disorders, 2 (2), 89-98.

25) Simons-Morton, B. G., Ouimet, M. C., Zhang, Z., Klauer, S. E., Lee, S. E., Wang, J., Chen, R., Albert, P., and Dingus, T. A. (2011) "The Effect of Passengers and Risk-Taking Friends on Risky Driving and Crashes/Near Crashes Among Novice Teenagers," Journal of Adolescent Health, 49, 587-593.

26) Stanford, M. S., Greeve, K. W., Boudreaux, J. K., Mathias, C. W., and Brumbelow, J. L. (1996) "Impulsiveness and risk-taking: Comparison of high-school and college students using the Barratt Impulsiveness Scale," Personality and Individual Differences, 21, 1073-1075.

27) Thomson, C. J., Morton, K. L., Carlson, S. R., and Rupert, J. L. (2012) "The Contextual Sensation Seeking Questionnaire for Skiing and Snowboarding (CSSQ-S) : Development of a sport specific scale," International Journal of Sport Psychology, 43, 503-521.

Linda Paquette, Maggie Dumais, Jacques Bergeron and Éric Lacourse (2016), Pediatrics Research International Journal, DOI: 10.5171/2016.405500 
28) Thorpe, H. (2004) "Embodied boarders: Snowboarding, status and style," Waikato Journal of Education, 10, 181-202.

29) Tremblay, R. E., Pihl, R. O., Vitaro, F., and Dobkin, P. L. (1994) "Predicting early onset of male antisocial behavior from preschool behavior," Archives of General Psychiatry, 51, 732-739.

30) Turner, C., McClure R, and Pirozzo S. (2004) "Injury and risk-taking behavior-a systematic review," Accident Analysis and Prevention, 36 (1), 93-101.

31) Woodman, T., Barlow, M., Bandura, C., Hill, M., Kupciw, D., and Macgregor, A.
(2013) " Not all risks are equal: the risktaking inventory for high-risk sports," Journal of Sports and Exercise Psychology, 35 (5), 479-492.

32) Xiang, H., Kelleher, K., Shields, B. J., Brown, K. J., and Smith, G. A. (2005) "Skiing- and snowboarding-related injuries treated in the U.S. emergency departments, 2002," The Journal of Trauma : injury, infection and critical care, 58, 112-118.

33) Zuckerman, M., and Kuhlman, D.M. (2000) "Personality and risk-taking: Common biosocial factors," Journal of Personality, 68 (6), 999-1029.

\section{Web references}

Snowsports Industries America (SIA). Snow Sports Fact Sheet. Available at: http://www.snowsports.org/SuppliersServiceProviders/Research/SnowSportsFactSheet. Accessed July 5, 2010. 